\title{
Quality and Damping Factors Optimization Using Taguchi Methods in Cantilever Beam Based Piezoelectric Micro-Power Generator for Cardiac Pacemaker Applications
}

\author{
Mohd H. S. Alrashdan
}

\begin{abstract}
Piezoelectric Micro-Power Generator (PMPG) can produce electrical power as a result of piezoelectric effects. Moreover, producing self-powering devices, especially in small electronic devices with low power requirements such as cardiac pacemakers is one of the objectives of the study. This paper optimizes the quality and damping factors for PMPG by using the Taguchi optimization method instead of a trial and error approach. Eight physical control parameters with three levels, including PMPG layer materials and dimensions are selected in order to study the influence of each parameter of PMPG quality and damping factors. Level eighteen Orthogonal arrays is based on signal-to-noise ratio which is conducted and confirmed by using analysis of variance in determining the PMPG with the best quality and damping factor. 18 experiments are conducted with three trials using COMSOL Multiphysics software ver. 5.4 for each one. The PMPG maximum quality factor, the lower damping factor, and the highest efficiency are designed at $1.35 \mathrm{~Hz}$, which is equivalent to 81 beats per min. Both Taguchi and the analysis of variance results conclude that the highest PMPG physical control parameters affect the quality and damping factors at $1.35 \mathrm{~Hz}$ from higher to lower order as follows: insulator width of $0.12 \mathrm{~mm}, 0.2 \mathrm{~mm}$ piezoelectric layer width, $20 \mu \mathrm{m}$ of insulator thickness, $2.5 \mathrm{~mm}$ of proof mass thickness, piezoelectric material of PZT5A, piezoelectric layer thickness of $60 \mu \mathrm{m}$, proof mass material of aluminum, and $5 \mathrm{~mm}$ proof mass length respectively. COMSOL Multiphysics is used again to study the PMPG with the best parameters, the PMPG resonates at $1.35 \mathrm{~Hz}, 5.78 \mathrm{~Hz}, 7.61$ $\mathrm{Hz}, 32.54 \mathrm{~Hz}, 424.21 \mathrm{~Hz}$, and $448.59 \mathrm{~Hz}$ respectively with a different mode of deflections by using Eigen's frequency analysis. The high electric field, the electric energy density, and hence the output power are produced at first resonance frequency at $1.35 \mathrm{~Hz}$ in the range of $0.5-2.5 \mathrm{~Hz}$ by using frequency response analysis. Transient analysis is conducted at $1.35 \mathrm{~Hz}$, which shows that the PMPG reaches the steady-state after seven seconds of sinusoidal signal excitation with quality factor of 166.67 and damping factor of 0.003. This improves that PMPG works nearly at no damping system and keeps oscillating for a long time before stopping for a single excitation, which opens the door widely in front of fabricating self-powered devices that have been working for a long time, which make them suitable to replace lithium iodide battery in cardiac pacemakers. Copyright $(2020$ The Authors.

Published by Praise Worthy Prize S.r.l. This article is open access published under the CC BY-NC-ND license (http://creativecommons.org/licenses/by-nc-nd/3.0/).
\end{abstract}

Keywords: Piezoelectric Micro Power Generator, Taguchi Method, MEMS Cantilever Beam, Quality Factor, Damping Factor, Eigen Frequency, Frequency Response, Transient Response Analysis

\section{Nomenclature}

$P_{\max } \quad$ Maximum Output Electrical Power

$m \quad$ Mass

Y Input Displacement

$\omega_{n} \quad$ Resonant Frequency

$\zeta \quad$ Damping Factor

$Q \quad$ Quality Factor

$\alpha \quad$ Mass Coefficients

$\beta \quad$ Stiffness Coefficients

$b \quad$ Unnamed Symbol used for calculation

$\omega_{1}$

$\begin{array}{ll}\omega_{2} & \text { Second Angular Resonance Frequency } \\ \text { PMPG } & \text { Piezoelectric Micro-Power Generator } \\ \text { OA } & \text { Orthogonal Arrays } \\ \text { L18 } & \text { Level Eighteen } \\ \text { S/N } & \text { Signal to Noise Ratio } \\ \text { ANOVA } & \text { Analysis Of Variance } \\ \text { MEMS } & \text { Micro-Electromechanical Systems } \\ \text { DOF } & \text { Degree of Freedom } \\ \text { AHSF } & \text { Abdul Hameed Shoman Foundation } \\ \text { SS } & \text { Sum of a Square } \\ \text { MS } & \text { Mean Square }\end{array}$




\section{Introduction}

The rapid improvement of the electronic devices in reducing their size, lowering the electric power needed, the rapid technology in improving material properties, and Micro-Electromechanical Systems (MEMS) technology opens the door in front of modelling and fabrication of self-powered devices by harvesting the available ambient power and converting it into usable electrical power [1]-[24]. This eliminates the need for replacing traditional batteries, since its replacement is difficult, costly, and needs medical surgery, especially in medical implants [1]-[4]. In the last two decades, the researchers have established power harvesting devices and fabricated it to power small electronic devices [5], based on the electrostatic method [6], electromagnetic [7] and piezoelectric effects [8]-[10], [22], [23]. The piezoelectric technique receives more attention, thanks to its easy fabrication process, which uses MEMS technology [11]. Piezoelectric Micro-Power Generator (PMPG) is simply an energy device that can harvest the available mechanical vibrational energy, then converts it into electrical energy via piezoelectric effects, which is normally fabricated by using MEMS technology [2], [12]. Different structures for PMPG including bridge, diaphragm, and Cantilever beam structure are employed in PMPG design [13]. The cantilever beam structure is favored over other structures in low resonance frequency applications below $100 \mathrm{~Hz}$ [8], [14]. Conversely, cantilever beam-based devices have a high correlation between their output power and physical parameter, such as dimensions, materials, loading, and vibration conditions [8] and [15]. This makes the PMPG design with the suitable damping factor, quality factor, and resonance frequency becomes very problematic. The PMPG output power can be characterized by its resonance frequency and damping constant [2], [8] as shown in equation (1):

$$
P_{\max }=\frac{m Y^{2} \omega_{n}^{3}}{4 \zeta}
$$

where $P_{\max }$ is the maximum output of electrical power produced, $m$ is the PMPG mass $(\mathrm{kg}), \zeta$ is the damping factor, $Y$ is an input displacement, and $\omega_{n}$ is the natural or resonant frequency $(\mathrm{rad} / \mathrm{s})$. All these parameters are sensitive to the device structure and the physical parameters. Like all the dynamic problems, the damping factor is very important in determining the output power of the PMPG. The PMPG damping factor is proportional to the mass $\alpha$ and to the stiffness coefficient $\beta$ in the following way [8]:

$$
\begin{gathered}
Q=\frac{\text { output displacement }}{\text { input displacement }} \\
\zeta=\frac{1}{2 Q}
\end{gathered}
$$

$$
\begin{gathered}
\alpha=\frac{\omega_{1}}{\omega_{2}} \\
b=\frac{(1-\alpha) \zeta}{\left(\omega_{2}-\alpha \omega_{1}\right)} \\
\beta=2 b
\end{gathered}
$$

where $\omega_{1}$ and $\omega_{2}$ are the first two angular resonance frequencies $(\mathrm{rad} / \mathrm{s})$, while $Q$ is the quality factor. A PMPG design problem for any application has many factors that affect its function including the ambient noise factors, device materials, and dimensions. Only few of these factors have the most significant effect on PMPG output power, while the other ones are negligible. Many experiments, longer time, and high cost are needed in designing PMPG using the trial and error method.

Taguchi, on the other hand is one of the most engineering optimization methods that can be used to determine the most relevant factor on desired output with a minimum number of experiments. In the Taguchi method, the number of experiments needed is minimized using specified Orthogonal Array (OA), which is based on the number of the control factors selected and the number of levels for each factor [16], [17]. By measuring the Signal to Noise ratio $(\mathrm{S} / \mathrm{N})$ for each experiment in the specified OA, is significant to find each control factor in the PMPG output power and to the optimum design that can be reached instead of testing all the possible variables in the trial and error method [18]. In this work, the PMPG model is selected, based on a previous study carried out in order to maximize the output power density [10], which is mainly composed of a cantilever beam structure with four fingers. Single-crystal silicon substrate is used as supportive material, silicon nitride layer is used as a buffer or barrier to prevent the lead migration from functioning piezoelectric materials into silicon substrate during the fabrication process, a layer of piezoelectric material can convert the mechanical vibration into electric power, a pair of electrodes is to collect the electric charge which is collected inside piezoelectric materials, and proof mass which is located at the cantilever beam free end for lowering the resonance frequency [10] as shown in Fig. 1. This study focuses mainly on optimizing the PMPG physical factors such as its dimensions, shape, and material type which affect quality factor, damping factor, and hence, the output power by using the Taguchi optimization method.

Furthermore, The COMSOL Multi-Physics simulation software is used to study the behaviors of the optimized PMPG model for the biomedical implant below $10 \mathrm{~Hz}$ ambient noise.

The following sections describe the methodology of this work including the working principles, followed by the results and the discussions of the main results obtained in this paper, based on proposed methodology.

Finally, the conclusion sums up the main results and objectives in this work. 


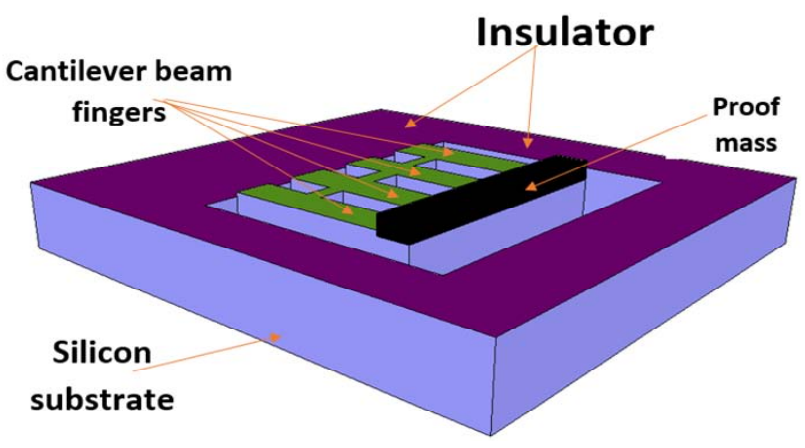

Fig. 1. PMPG four finger comp shape cantilever beam structure

\section{Methodology}

Taguchi's optimization method instead of trial and error is implemented in this work in order to select the most control factors affect PMPG damping and quality factors. Eight control factors with zero noise level are selected based on a study carried to optimize the PMPG output power density for cardiac pacemakers [8], [10].

These control factors with three levels are shown in Table I [10], where the other parameters are kept constant. L18 orthogonal array was used as illustrated in Table II with 17 Degree of Freedom (DOF), assigned as two DOFs for each factor except the proof mass material with one DOF, and two DOFs for error [16]. The device dimensions and materials are chosen in order to meet the available MEMS fabrication technology in Jordan [19], [20]. The PMPG length and width are kept constant to $30.4 \mathrm{~mm}$ (X-axis) and $19.05 \mathrm{~mm}$ (Y-axis) respectively, while the insulation material is chosen to be silicon nitride $\left(\mathrm{Si}_{3} \mathrm{~N}_{4}\right)$ due to its compatibility with the silicon substrate [21]. Practically, many noises factors affect device performance, such as temperature variation, other electromagnetic signals from other devices, atmospheric pressure difference, and velocity. It is assumed that the noise factors affect the control factor with the same percentage does not affect the order control factor in determining the damping and quality factor in PMPG.

Therefore, in the PMPG simulation study, the PMPG device is assumed to be without noise factors. COMSOL Multiphysics ver. 5.4 is used for 18 experiment simulations: the first and the second resonance Eigen frequency $(\mathrm{Hz})$ are equal to the first and the second angular resonance frequencies, $\omega_{1}$ and $\omega_{2}$ are found. The total displacement is measured at the center of the proof mass in the first and the second resonance Eigen frequency.

TABLE I

PMPG CONTROL PARAMETERS AND ITS LEVELS

\begin{tabular}{cccc}
\hline \hline Parameter & Level 1 & Level 2 & Level 3 \\
\hline Proof mass material (P1) & Aluminum & Gold & SU8 \\
Piezoelectric material (P2) & Gallium Arsenide & Pzt-5a & Pzt-5h \\
Proof mass length (P3) & $3 \mathrm{~mm}$ & $5 \mathrm{~mm}$ & $7 \mathrm{~mm}$ \\
Proof mass thickness (P4) & $1 \mathrm{~mm}$ & $2.5 \mathrm{~mm}$ & $4 \mathrm{~mm}$ \\
Piezo width (P5) & $0.12 \mathrm{~mm}$ & $0.16 \mathrm{~mm}$ & $0.2 \mathrm{~mm}$ \\
piezo thickness (P6) & $30 \mu \mathrm{m}$ & $45 \mu \mathrm{m}$ & $60 \mu \mathrm{m}$ \\
Insulator width (P7) & $0.12 \mathrm{~mm}$ & $0.16 \mathrm{~mm}$ & $0.2 \mathrm{~mm}$ \\
Insulator thickness (P8) & $20 \mu \mathrm{m}$ & $30 \mu \mathrm{m}$ & $40 \mu \mathrm{m}$ \\
\hline \hline
\end{tabular}

TABLE II

TAGUCHI L18 ORTHOGONAL ARRAY

\begin{tabular}{ccccccccc}
\multicolumn{7}{c}{ TAGUCHI L18 ORTHOGONAL ARRAY } \\
\hline \hline Exp & P1 & P2 & P3 & P4 & P5 & P6 & P7 & P8 \\
\hline 1 & 1 & 1 & 1 & 1 & 1 & 1 & 1 & 1 \\
2 & 1 & 1 & 2 & 2 & 2 & 2 & 2 & 2 \\
3 & 1 & 1 & 3 & 3 & 3 & 3 & 3 & 3 \\
4 & 1 & 2 & 1 & 1 & 2 & 2 & 3 & 3 \\
5 & 1 & 2 & 2 & 2 & 3 & 3 & 1 & 1 \\
6 & 1 & 2 & 3 & 3 & 1 & 1 & 2 & 2 \\
7 & 1 & 3 & 1 & 2 & 1 & 3 & 2 & 3 \\
8 & 1 & 3 & 2 & 3 & 2 & 1 & 3 & 1 \\
9 & 1 & 3 & 3 & 1 & 3 & 2 & 1 & 2 \\
10 & 2 & 1 & 1 & 3 & 3 & 2 & 2 & 1 \\
11 & 2 & 1 & 2 & 1 & 1 & 3 & 3 & 2 \\
12 & 2 & 1 & 3 & 2 & 2 & 1 & 1 & 3 \\
13 & 2 & 2 & 1 & 2 & 3 & 1 & 3 & 2 \\
14 & 2 & 2 & 2 & 3 & 1 & 2 & 1 & 3 \\
15 & 2 & 2 & 3 & 1 & 2 & 3 & 2 & 1 \\
16 & 2 & 3 & 1 & 3 & 2 & 3 & 1 & 2 \\
17 & 2 & 3 & 2 & 1 & 3 & 1 & 2 & 3 \\
18 & 2 & 3 & 3 & 2 & 1 & 2 & 3 & 1 \\
\hline \hline
\end{tabular}

Then the quality factor, the damping factor and the related damping parameters including mass $\alpha$ and stiffness $\beta$ coefficients are calculated for each experiment.

This simulation is repeated three times for each experiment. Each one is conducted with three different meshing scales geometry as shown in Table III.

MINITAB 17 software is used to analyze the data based on Taguchi tools, which is considered as the most influential control factor that affects the first and the second resonance Eigen frequency and hence, the quality and damping factor of PMPG based on the S/N ratio with the concept of "the larger-the-better" is found [16]. This technique has brought down the number of the factors that are the most influential in PMPG performance for biomedical implants at extremely low frequency below $10 \mathrm{~Hz}$. COMSOL Multiphysics 5.4 software is used for three basic analyses, including Eigen frequency, frequency response, and Transient analysis of PMPG device with optimum control parameters. During free vibration, PMPG operates at more than one resonant frequency.

The purpose of the Eigen frequency analysis is to find the lowest six resonance frequencies and their subsequent shape modes, in order to find the most suitable modes of operation for PMPG during vibration. The frequency response analysis is used to find the PMPG displacement in all the directions, which are electric field, Von Mises stress, Tresca stresses, and PMPG electric energy density in frequency ranges covers the first four resonance frequencies. The transient analysis is used to study the device that produces voltage, electric charge density, and PMPG displacement as functions of time during vibration.

TABLE III

PMPG MESHING SCALE GEOMETRY

\begin{tabular}{cccc}
\hline \hline Factor & Trial 1 & Trial 2 & Trial 3 \\
\hline X-Direction factor & 1.0 & 0.5 & 0.1 \\
Y-Direction factor & 1.0 & 0.5 & 0.1 \\
Z-direction scale factor & 20 & 10 & 1 \\
Resolution of geometry & 20 & 10 & 1 \\
\hline \hline
\end{tabular}




\section{Results and Discussion}

Tables IV, V, and VI below show the simulation results of 18 experiments for three different trials. They represent the total displacements at the first and the second resonance frequency, quality factor, mass $\alpha$, stiffness $\beta$ coefficients and the damping factor. The damping factor reduces the PMPG from vibration, which lowers the output power generation. The damping factor for all eighteen experiments with three trials is less than 0.3 , whereas all the systems are considered underdamped except experiment number 9 in trial number three. In these experiments the PMPG vibrates and produces the output power as a result of vibration at a reduced frequency, where the damping factor is higher than 1.9 since the total displacement at the second mode of operation is higher than the first mode of operation. The system of experiment number 9 is considered overdamped, then it returns to equilibrium without oscillation while the produced power is minimum. It is clear that the all the damping factors for all the experiments except number 9 are approximately zero, where the system oscillates exactly at the natural resonance frequency continuously without restriction. In reality, the damping factor is not exactly zero and there is a life cycle of oscillation, but since the ambient mechanical vibration is available; the PMPG can work in an infinite life span.

Fig. 2 and Table VII show signal to noise ratio $(\mathrm{S} / \mathrm{N})$ and how each control parameter affects the damping factor using Taguchi method along with Minitab 17 statistical software. The optimal level of PMPG damping factor and therefore less output power generation are selected to aluminum proof mass, PZT-5A piezoelectric material, 3- $\mathrm{mm}$ proof mass, length, $4 \mathrm{~mm}$ proof mass thickness, $0.12-\mathrm{mm}$ piezoelectric layer width, $60 \mu \mathrm{m}$ piezoelectric layer thickness, $0.16 \mathrm{~mm}$ Insulator width, and $40 \mu \mathrm{m}$ insulator thickness. With these values, the damping factor will be the maximum and the output power generator will be at the lowest level.

TABLE IV

PMPG SIMULATION QUALITY AND DAMPING FACTOR RESULTS FOR TRIAL 1

\begin{tabular}{|c|c|c|c|c|c|c|c|c|}
\hline Exp Num & $\begin{array}{l}\text { 1st resonance } \\
\text { frequency }(\mathrm{Hz})\end{array}$ & $\begin{array}{l}\text { 2nd resonance } \\
\text { frequency }(\mathrm{Hz})\end{array}$ & $\begin{array}{l}\text { Total displacement } \\
1 \text { st mode }(\mu \mathrm{m})\end{array}$ & Total displacement 2 nd mode $(\mu \mathrm{m})$ & Q & $\zeta$ & $\alpha$ & $\beta$ \\
\hline 1 & 1.09 & 5 & 165 & 3.5 & 47.14286 & 0.010606 & 0.218 & 0.000554 \\
\hline 2 & 1.22 & 5.44 & 19 & 8.5 & 2.235294 & 0.223684 & 0.224265 & 0.010691 \\
\hline 3 & 2.93 & 13.79 & 160 & 79 & 2.025316 & 0.246875 & 0.212473 & 0.0047 \\
\hline 4 & 2.97 & 14.05 & 7 & 0.38 & 18.42105 & 0.027143 & 0.211388 & 0.000508 \\
\hline 5 & 1.35 & 5.78 & 300 & 1.8 & 166.6667 & 0.003 & 0.233564 & 0.000134 \\
\hline 6 & 0.54 & 2.45 & 100 & 14 & 7.142857 & 0.07 & 0.220408 & 0.007452 \\
\hline 7 & 2.13 & 7.54 & 11 & 4.5 & 2.444444 & 0.204545 & 0.282493 & 0.006733 \\
\hline 8 & 0.57 & 3.01 & 40 & 6.5 & 6.153846 & 0.08125 & 0.189369 & 0.007224 \\
\hline 9 & 2.37 & 11.39 & 0.001 & .005 & 0.2 & 2.5 & 0.208077 & 0.057832 \\
\hline 10 & 2.72 & 11.59 & 71 & 40 & 1.775 & 0.28169 & 0.234685 & 0.006266 \\
\hline 11 & 7.93 & 31.66 & .012 & .0025 & 4.8 & 0.104167 & 0.250474 & 0.000838 \\
\hline 12 & 2.41 & 10.26 & 130 & 14.5 & 8.965517 & 0.055769 & 0.234893 & 0.001401 \\
\hline 13 & 4.06 & 20.27 & 69 & 33 & 2.090909 & 0.23913 & 0.200296 & 0.003129 \\
\hline 14 & 2.59 & 8.76 & 110 & 40 & 2.75 & 0.181818 & 0.295662 & 0.005099 \\
\hline 15 & 4.72 & 20.83 & 36 & 6.2 & 5.806452 & 0.086111 & 0.226596 & 0.001073 \\
\hline 16 & 4.03 & 14.06 & 39 & 17.75 & 2.197183 & 0.227564 & 0.286629 & 0.004004 \\
\hline 17 & 5.06 & 25.37 & 25 & 0.65 & 38.46154 & 0.013 & 0.199448 & 0.000136 \\
\hline 18 & 2.24 & 11.41 & 280 & 7 & 40 & 0.0125 & 0.196319 & 0.000291 \\
\hline
\end{tabular}

TABLE V

PMPG SIMULATION QUALITY AND DAMPING FACTOR RESULTS FOR TRIAL 2

\begin{tabular}{|c|c|c|c|c|c|c|c|c|}
\hline $\begin{array}{l}\text { Exp } \\
\text { Num }\end{array}$ & $\begin{array}{l}\text { 1st resonance } \\
\text { frequency }(\mathrm{Hz})\end{array}$ & $\begin{array}{l}\text { 2nd resonance } \\
\text { frequency }(\mathrm{Hz})\end{array}$ & $\begin{array}{c}\text { Total displacement } \\
1 \text { st mode }(\mu \mathrm{m})\end{array}$ & Total displacement 2 nd mode $(\mu \mathrm{m})$ & $Q$ & $\zeta$ & $\alpha$ & $\beta$ \\
\hline 1 & 1.10 & 4.88 & 110 & 1.9 & 57.89474 & 0.008636 & 0.22541 & 0.00046 \\
\hline 2 & 1.23 & 5.72 & 21 & 3.3 & 6.363636 & 0.078571 & 0.215035 & 0.003599 \\
\hline 3 & 2.87 & 13.32 & 210 & 37 & 5.675676 & 0.088095 & 0.215465 & 0.001732 \\
\hline 4 & 2.96 & 14.14 & 8 & 1.2 & 6.666667 & 0.075 & 0.209335 & 0.001396 \\
\hline 5 & 1.35 & 5.71 & 170 & 5.5 & 30.90909 & 0.016176 & 0.236427 & 0.000729 \\
\hline 6 & 0.58 & 3.12 & 65 & 10 & 6.5 & 0.076923 & 0.185897 & 0.006618 \\
\hline 7 & 2.13 & 7.72 & 10 & 4.6 & 2.173913 & 0.23 & 0.275907 & 0.007433 \\
\hline 8 & 0.57 & 3.04 & 37 & 5.2 & 7.115385 & 0.07027 & 0.1875 & 0.006196 \\
\hline 9 & 2.38 & 11.39 & 0.0009 & 0.0035 & 0.257143 & 1.944444 & 0.208955 & 0.044948 \\
\hline 10 & 2.72 & 11.57 & 56 & 6.2 & 9.032258 & 0.055357 & 0.235091 & 0.001233 \\
\hline 11 & 8.06 & 31.97 & 0.018 & 0.0021 & 8.571429 & 0.058333 & 0.252111 & 0.000464 \\
\hline 12 & 2.43 & 10.70 & 390 & 160 & 2.4375 & 0.205128 & 0.227103 & 0.004973 \\
\hline 13 & 4.08 & 20.13 & 20 & 4.3 & 4.651163 & 0.1075 & 0.202683 & 0.001413 \\
\hline 14 & 2.59 & 8.79 & 80 & 26 & 3.076923 & 0.1625 & 0.294653 & 0.004545 \\
\hline 15 & 4.93 & 23.09 & 33 & 17 & 1.941176 & 0.257576 & 0.213512 & 0.002926 \\
\hline 16 & 4.37 & 18.10 & 36 & 16 & 2.25 & 0.222222 & 0.241436 & 0.003148 \\
\hline 17 & 5.05 & 25.47 & 46 & 0.6 & 76.66667 & 0.006522 & 0.198272 & $6.8 \mathrm{E}-05$ \\
\hline 18 & 2.24 & 11.29 & 150 & 16 & 9.375 & 0.053333 & 0.198406 & 0.001255 \\
\hline
\end{tabular}


TABLE VI

PMPG SIMULATION QUALITY AND DAMPING FACTOR RESULTS FOR TRIAL 3

\begin{tabular}{|c|c|c|c|c|c|c|c|c|}
\hline $\begin{array}{l}\text { Exp } \\
\text { Num }\end{array}$ & $\begin{array}{c}\text { 1st resonance } \\
\text { frequency }(\mathrm{Hz})\end{array}$ & $\begin{array}{l}\text { 2nd resonance } \\
\text { frequency }(\mathrm{Hz})\end{array}$ & $\begin{array}{l}\text { Total displacement } \\
1 \text { st mode }(\mu \mathrm{m})\end{array}$ & Total displacement 2 nd mode $(\mu \mathrm{m})$ & $Q$ & $\zeta$ & $\alpha$ & $\beta$ \\
\hline 1 & 1.08 & 4.76 & 19 & 3.7 & 5.135135 & 0.097368 & 0.226891 & 0.005307 \\
\hline 2 & 1.18 & 5.12 & 24 & 4.0 & 6 & 0.083333 & 0.230469 & 0.00421 \\
\hline 3 & 2.66 & 11.19 & 270 & 35 & 7.714286 & 0.064815 & 0.237712 & 0.00149 \\
\hline 4 & 2.93 & 13.44 & 16 & 0.6 & 26.66667 & 0.01875 & 0.218006 & 0.000365 \\
\hline 5 & 1.33 & 5.55 & 19 & 1.5 & 12.66667 & 0.039474 & 0.23964 & 0.001826 \\
\hline 6 & 0.54 & 2.34 & 73 & 10.5 & 6.952381 & 0.071918 & 0.230769 & 0.007949 \\
\hline 7 & 2.12 & 7.57 & 9.7 & 4.2 & 2.309524 & 0.216495 & 0.280053 & 0.007112 \\
\hline 8 & 0.56 & 2.87 & 80 & 3.5 & 22.85714 & 0.021875 & 0.195122 & 0.00203 \\
\hline 9 & 2.22 & 10.30 & 0.0008 & 0.035 & 0.022857 & 21.875 & 0.215534 & 0.556152 \\
\hline 10 & 2.68 & 11.14 & 60 & 8 & 7.5 & 0.066667 & 0.240575 & 0.001536 \\
\hline 11 & 7.59 & 28.93 & 0.011 & 0.0045 & 2.444444 & 0.204545 & 0.262357 & 0.001783 \\
\hline 12 & 2.41 & 10.38 & 115 & 10.5 & 10.95238 & 0.045652 & 0.232177 & 0.001136 \\
\hline 13 & 3.66 & 16.53 & 52 & 3.4 & 15.29412 & 0.032692 & 0.221416 & 0.000515 \\
\hline 14 & 2.56 & 8.54 & 220 & 34 & 6.470588 & 0.077273 & 0.299766 & 0.002216 \\
\hline 15 & 4.77 & 21.30 & 35 & 29 & 1.206897 & 0.414286 & 0.223944 & 0.005058 \\
\hline 16 & 4.50 & 16.55 & 42 & 6.5 & 6.461538 & 0.077381 & 0.271903 & 0.00117 \\
\hline 17 & 5.00 & 24.40 & 31 & 1.1 & 28.18182 & 0.017742 & 0.204918 & 0.000192 \\
\hline 18 & 2.22 & 10.96 & 115 & 28.5 & 4.035088 & 0.123913 & 0.202555 & 0.002993 \\
\hline
\end{tabular}

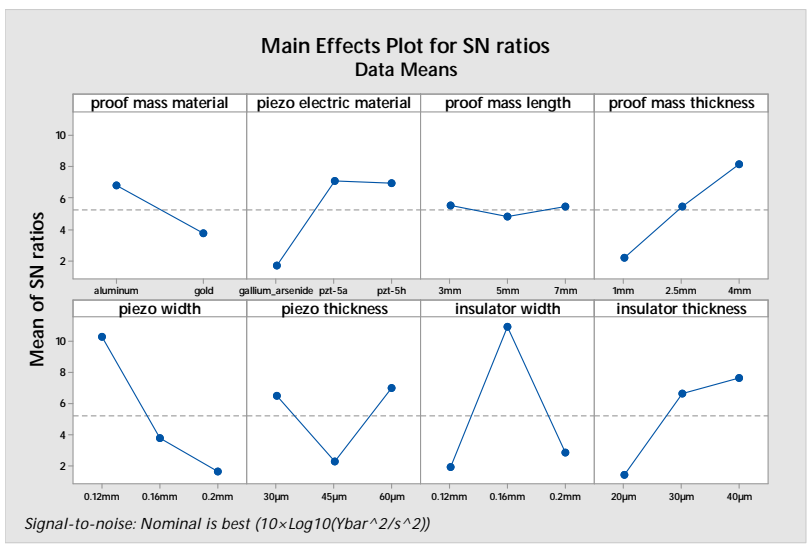

Fig. 2. Effect of the control parameters with the PMPG damping factor values for the $\mathrm{S} / \mathrm{N}$ ratio

Table VII ranks the control parameters', by effecting on its damping factor in descending order from 1-8 based on signal to noise ratio, where the insulator width control parameters have the biggest influence in PMPG damping factor, followed by piezoelectric layer width, insulator thickness, proof mass thickness, piezoelectric material, piezoelectric layer thickness, proof mass material, and finally proof mass length control factor. Therefore, any change in control factor, which has a higher rank, such as insulator width, leads to a higher effect of damping factor in comparison to the factors that have a low rank such as proof mass length. Ideally, the PMPG design needs to work with no damping coefficients, i.e. $\zeta$ equals zero in order to confirm the PMPG, which works only at the resonance frequency and oscillates or vibrates without stopping. In reality, there is a frequency range for any mechanical design that can vibrate within this range and keep the maximum output power at the resonance frequency. Therefore, the optimum real design needs to have a very low damping factor, improving the PMPG oscillation for longer time under single excitation. The PMPG keeps working with the excitation source or any mechanical vibration that is available in the environment.

Therefore, the maximum output power generation, needs to concentrate on the control factor with less effect on damping factor, such as proof mass length. Based on this design, the mass of the proposed PMPG is mainly dominated by the proof mass, the width of the proof mass is kept constant at $19.05 \mathrm{~mm}$. Therefore, the length of the proof mass has the main effect on PMPG total mass, by reducing the cantilever beam resonance frequency for low-frequency range, and by maximizing output power generation. Proof mass material control factor also has a significant role in PMPG total mass, where the aluminum material is less in density compared with gold, the aluminum effects on the higher damping factor with less output power generation in comparison with gold. These control factors are followed by piezoelectric control factors, such as piezoelectric materials and their thickness. The highest factor affects the damping coefficients and leads to produce the smaller output power generation, such as insulator width, and insulator thickness, which has only a role in preventing Lead's movement from piezoelectric materials to substrate materials during fabrication process as a buffer layer.

Therefore, the major work for the insulating layer is considered as pure electrically [8]. Table VIII represents the analysis of damping factor variance of the ANOVA test for $95 \%$ significant confidence interval. The results show a double Degree of Freedom (DOF) for all the control variables except the proof mass material control variable, which has single DOF, related to Taguchi method orthogonal array L18, where DOF for each control variable is equivalent to the number of levels used for each control variable minus one in L18, as mentioned before in Table II.

TABLE VII

RESPONSE TABLE FOR SignAL TO NOISE RATIOS NOMINAL IS BEST $\left(10 \times \log 10\left(\mathrm{Ybar}^{\wedge} 2 / \mathrm{s}^{\wedge} 2\right)\right)$

\begin{tabular}{ccccccccc}
\hline \hline Level & $\mathrm{P}(1)$ & $\mathrm{P}(2)$ & $\mathrm{P}(3)$ & $\mathrm{P}(4)$ & $\mathrm{P}(5)$ & $\mathrm{P}(6)$ & $\mathrm{P}(7)$ & $\mathrm{P}(8)$ \\
\hline 1 & $6.7^{*}$ & 1.6 & $5.5^{*}$ & 2.1 & $10.3^{*}$ & 6.4 & 1.9 & 1.4 \\
2 & 3.7 & $7.1^{*}$ & 4.7 & 5.4 & 3.7 & 2.2 & $10.9^{*}$ & 6.6 \\
3 & & 6.9 & 5.4 & $8.1^{*}$ & 1.6 & $7.0^{*}$ & 2.8 & $7.6^{*}$ \\
Delta & 3.0 & 5.4 & 0.7 & 6.0 & 8.6 & 4.7 & 9.0 & 6.1 \\
Rank & 7 & 5 & 8 & 4 & 2 & 6 & 1 & 3 \\
\hline *.
\end{tabular}


For example, there are only two levels in this work for proof mass_material, which is aluminum and gold, so this control variable has a single DOF while all the other control variables have two DOFs. The total DOFs for PMPG in this work are 17 including two DOFs for the residual error. The Sum Squares (SS) and Mean Square (MS) value are shown in Table VIII, confirming the Taguchi results in Table VII, where the percentage of the control variable affects the quality and damping factors. This percentage is based on the ratio between the square sums for each control variable to the total square sum. It shows that insulator layer width has the higher percentage equal to $28.19 \%$, followed by piezoelectric layer width control variable with $23.24 \%$ and so on. The only mismatch between ANOVA test and Taguchi results is in the interchange between piezoelectric material and proof mass thickness. Taguchi experiment results show slightly a higher effect of proof mass thickness (rank 4) where piezoelectric material awkward (rank 5), while in ANOVA test piezoelectric material has a percentage of 10.92 and proof mass thickness has $10.32 \%$. This is a very slight difference between the two results that leads to conclude that both Taguchi and ANOVA support one another. COMSOL Multiphysics 5.4 3D MEMS module - structural mechanics - Piezo solid uses finite element method in order to simulate and study PMPG with dimensions produce the best quality factor and the lowest damping factor which is in the first trial experiment number five, whereas the quality factor equals to 166 and the damping factor is 0.003 , the PMPG vibrates at underdamped system, and it cannot be considered as damped system, whereas the damping factor is approximately equals to zero. This proves that the system can oscillate for a long time under a single excitation before stopping and can produce a continues electric charge once vibrates at the resonance frequency. Three basic analyses which are Eigen frequency, Frequency response, and Transient analysis are used in studying the PMPG device.

PMPG mesh statistics show 60194 degrees of freedom, 2995 mesh point, 8478 of tetrahedral elements, 6151 triangular boundary elements, 1838 number of edge elements, 71 vertex elements, 0.0105 minimum element quality, and $4.01 \times 10^{-7}$ element of volume ratio. Eigen frequency analysis used to find the first four modes of operation is shown in Fig. 3(a).

TABLE VIII

ANALYSIS OF VARIANCE (ANOVA) FOR SN RATIOS FOR A SIGNIFICANT LEVEL OF $95 \%$

\begin{tabular}{cccccccc}
\multicolumn{7}{c}{ FOR A SIGNIFICANT LEVEL OF 95 \% } \\
\hline \hline Source & DOF & Seq SS & Adj SS & Adj MS & F & P & $\%$ \\
\hline $\mathrm{P}(1)$ & 1 & 41.7 & 41.7 & 41.7 & 2.7 & 0.2 & 3.9 \\
$\mathrm{P}(2)$ & 2 & 114.9 & 114.9 & 57.4 & 3.8 & 0.2 & 10.9 \\
$\mathrm{P}(3)$ & 2 & 1.8 & 1.8 & 0.9 & 0.1 & 0.9 & 0.17 \\
$\mathrm{P}(4)$ & 2 & 108.4 & 108.4 & 54.2 & 3.5 & 0.2 & 10.3 \\
$\mathrm{P}(5)$ & 2 & 244.5 & 244.5 & 122.2 & 8.0 & 0.1 & 23.2 \\
$\mathrm{P}(6)$ & 2 & 80.7 & 80.7 & 40.3 & 2.6 & 0.3 & 7.68 \\
$\mathrm{P}(7)$ & 2 & 296.6 & 296.6 & 148.3 & 9.8 & 0.1 & 28.1 \\
$\mathrm{P}(8)$ & 2 & 133.0 & 133.0 & 66.5 & 4.4 & 0.2 & 12.6 \\
Residual & 2 & 30.2 & 30.2 & 15.1 & & & 2.87 \\
Error & 17 & 1052.13 & & & & & 100 \\
Total & 17
\end{tabular}

In the first mode, the PMPG resonates at $1.35 \mathrm{~Hz}$ and vibrates up and down in $\mathrm{Z}$ direction.

In the other ones as shown in Figs. 3(b)-(d), the PMPG shows a twist or another movement rather than up and down movement.

The neutral axis where the cantilever beam does not show compression or tension during vibration is located in the proof mass layer or outside the piezoelectric functioning layer, while this is very important to prevent the charge cancellation inside the piezoelectric material during vibration.

The first mode is preferable to the other ones in order to prevent charge cancellation inside the functioning piezoelectric materials during vibration. Since all the electric charges are collected at the electrode, they could be positive below the neutral axis during compression or they could be negative charge above the neutral axis during tension.

In the other mode like the twisting one shown in Figs. 3(b)-(d), the charge cancellation occurs due to compression and tension, which happens at the same time inside the piezoelectric materials. Furthermore, the first mode is preferred due to higher displacement, higher strain produced, and also higher output electric power produced within PMPG.

In frequency response analysis, the PMPG is simulated in the frequency range to cover the first four modes of operation from [0-50] Hz. Without applying any mechanical load to the PMPG device as with Eigen frequency, the PMPG vibrates at the resonance frequency due to its weight.

Figs. 4(a)-(d) show the PMPG frequency response displacement analysis.

Fig. 4(a) shows the PMPG total displacement measured at the center of the proof mass, while the maximum displacement occurs in the third mode of operation at $7.61 \mathrm{~Hz}$ followed by the first mode of operation at $1.35 \mathrm{~Hz}$, the total displacement at the third mode is dominated by $\mathrm{Z}, \mathrm{X}$, and $\mathrm{Y}$ displacements shown in Figs. 4 (b)-(d), respectively, where the PMPG vibrates in the twisting mode.

Conversely, the total displacement in the first mode is mainly dominated in $\mathrm{Z}$-axis displacement where the $\mathrm{X}$ and $\mathrm{Y}$-axis displacements are negligible; this produces higher normal electric field and higher energy density at the first mode of operation at $1.35 \mathrm{~Hz}$ in comparison with other resonance frequencies or modes of operations as shown in Figs. 5(a) and (b). Figs. 5(c) and (d) show the PMPG Tresca's stresses and Von Mises stress respectively.

The Tresca's stresses are used for determining the maximum stress of a material before yielding or fracture, while the Von Mises stress is used to predict yielding of PMPG under complex loading from the results of uniaxial tensile tests.

The PMPG will yield or fracture at about $0.6 \mathrm{MPa}$ of normal and shear stresses at the first resonance mode, which is suitable for bio implant devices, while it is up to 3.5 $\mathrm{MPa}$ for the third resonance mode. 


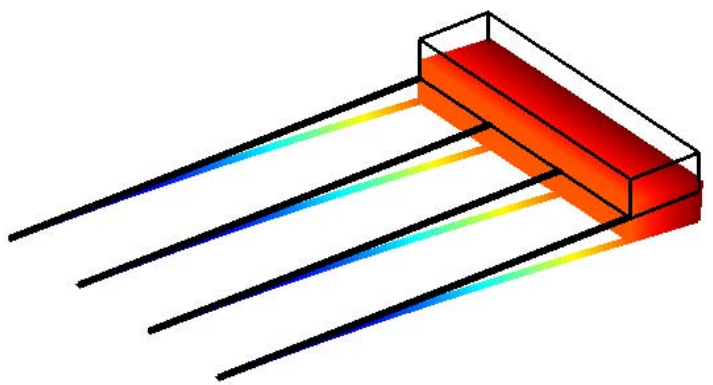

(a)

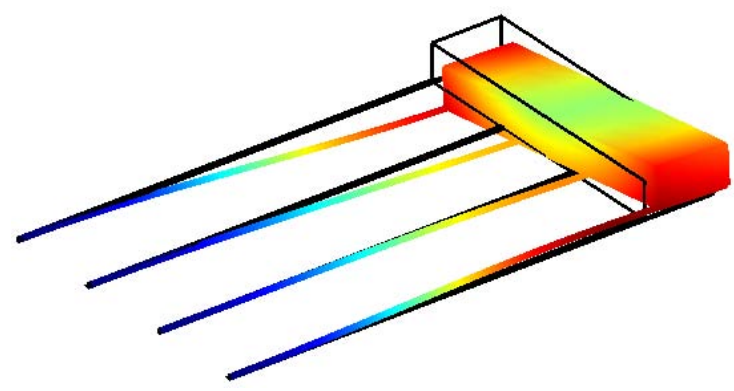

(c)

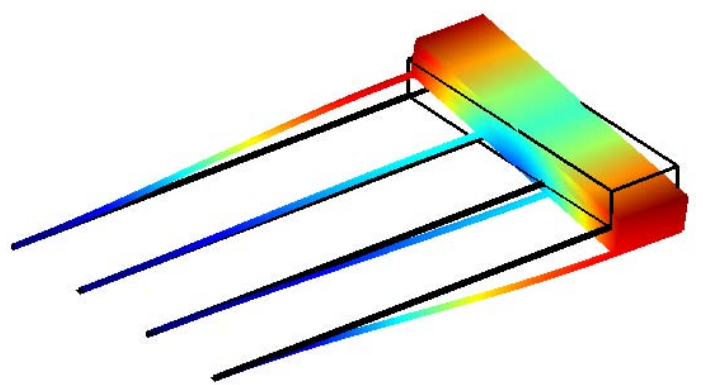

(b)

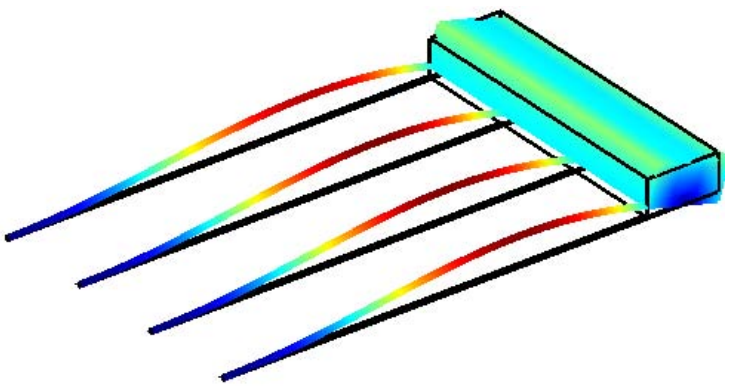

(d)

Figs. 3. PMPG deflections in the first four modes of operation: (a) 1st resonance mode at $1.35 \mathrm{~Hz}$, (b) 2nd resonance mode at $5.78 \mathrm{~Hz}$, (c) $3 \mathrm{ed}$ resonance mode at $7.61 \mathrm{~Hz}$, (d) 4 th resonance mode at $32.54 \mathrm{~Hz}$

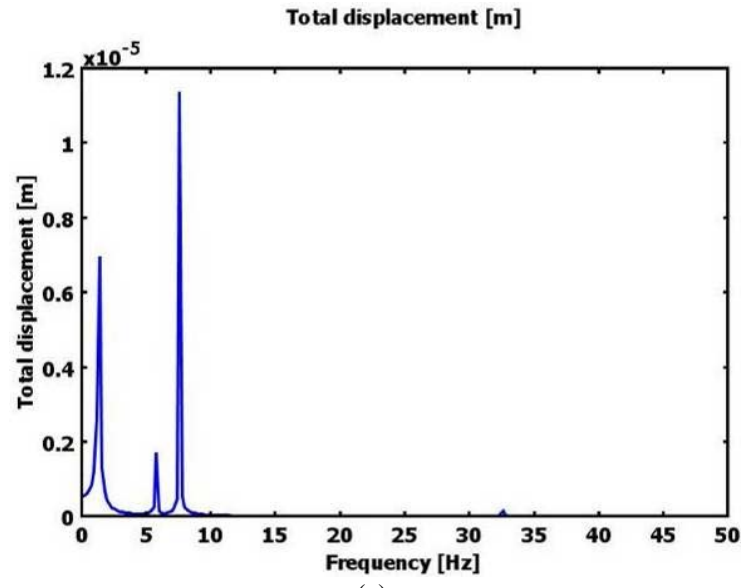

(a)

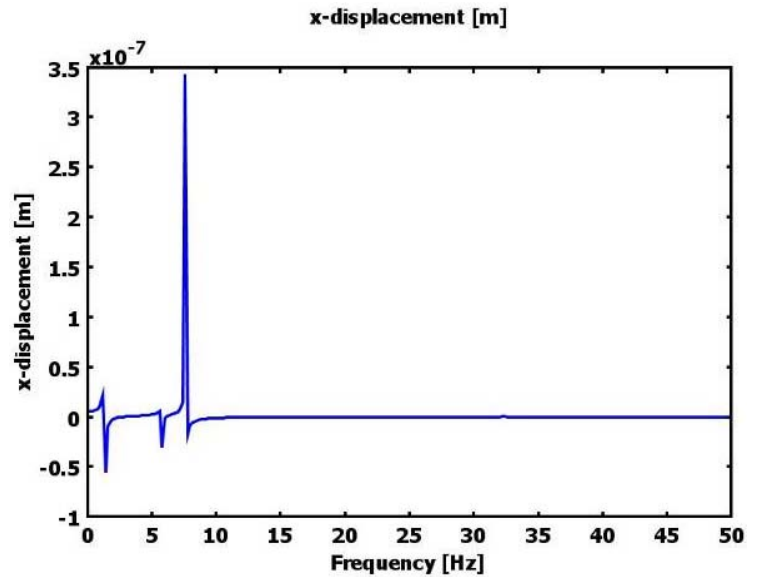

(c)

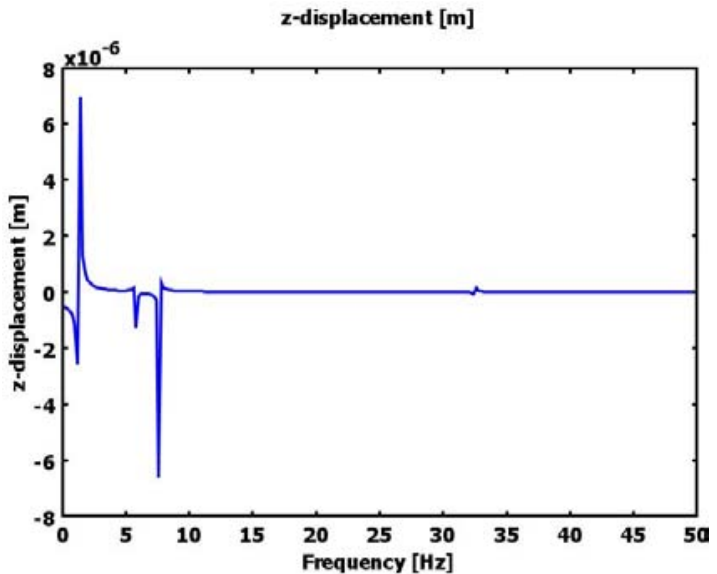

(b)

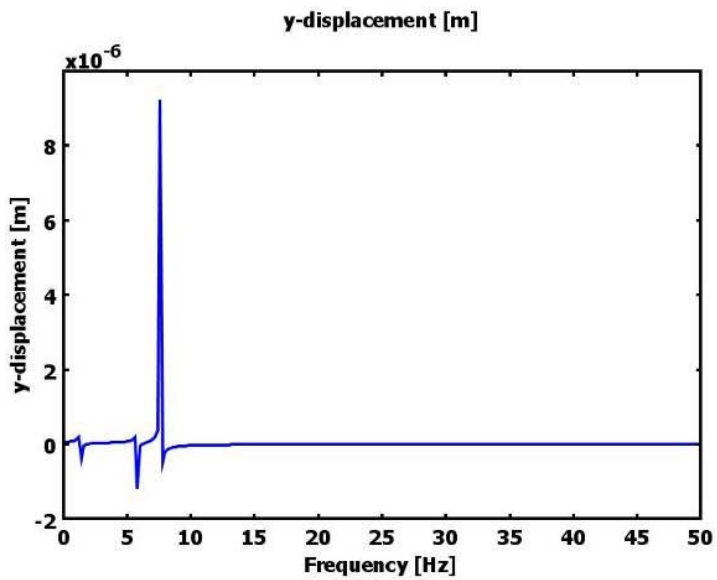

(d)

Figs. 4. PMPG Frequency response displacement analysis in range 0-50Hz:

(a) Total displacement, (b) Z displacement, (c) X displacement, (d) Y displacement 


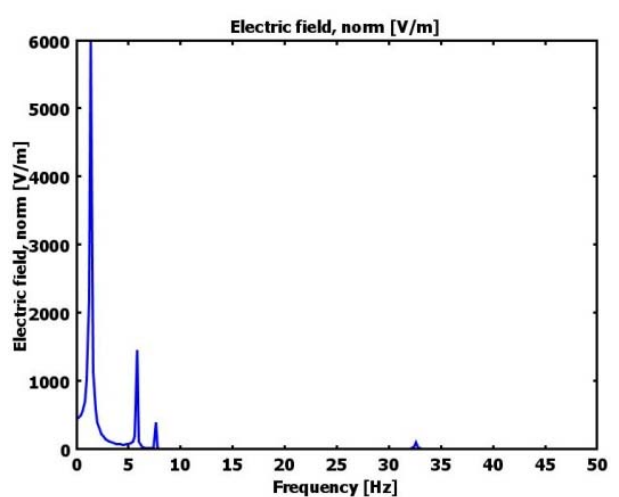

(a)

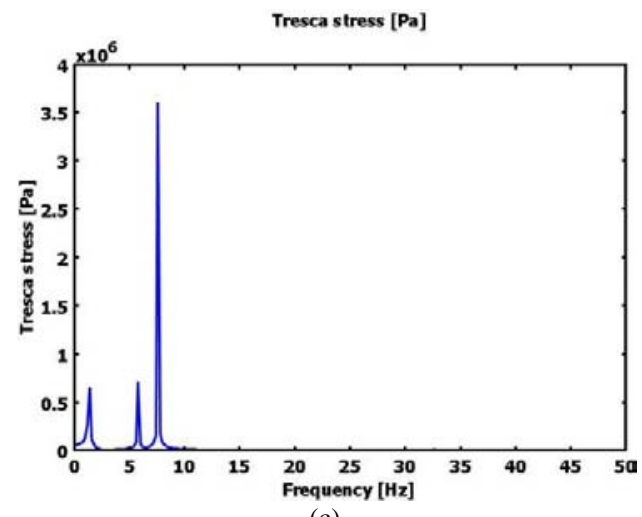

(c)

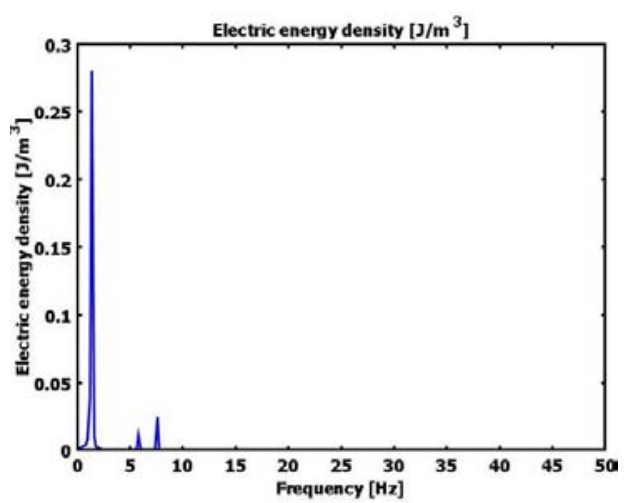

(b)

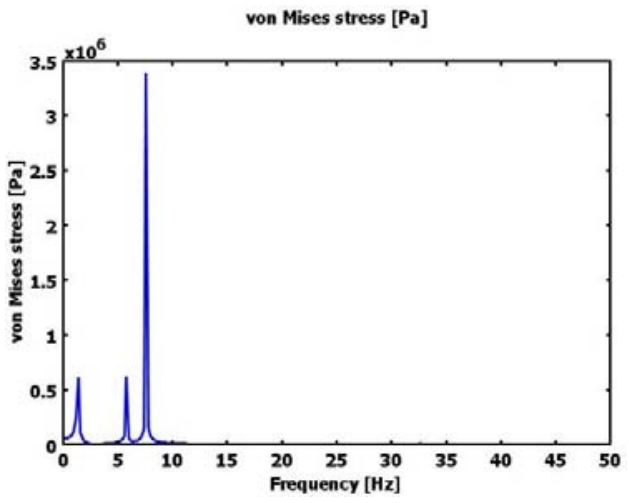

(d)

Figs. 5. PMPG Frequency response analysis in range 0-50 Hz:

(a) Normal electric field, (b) electric energy density, (c) Tresca stress, (d) Von Mises stress

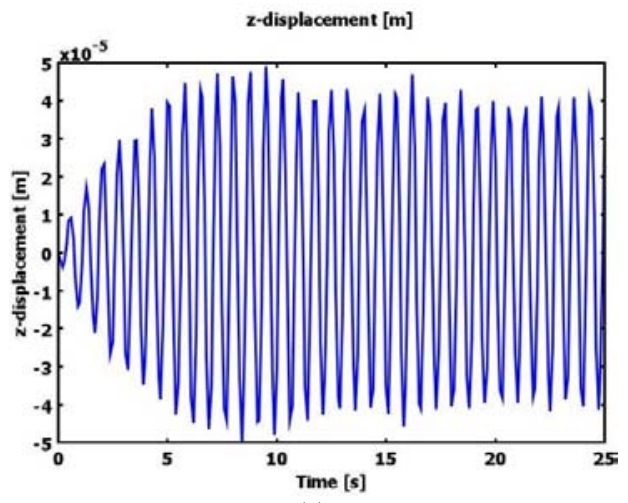

(a)

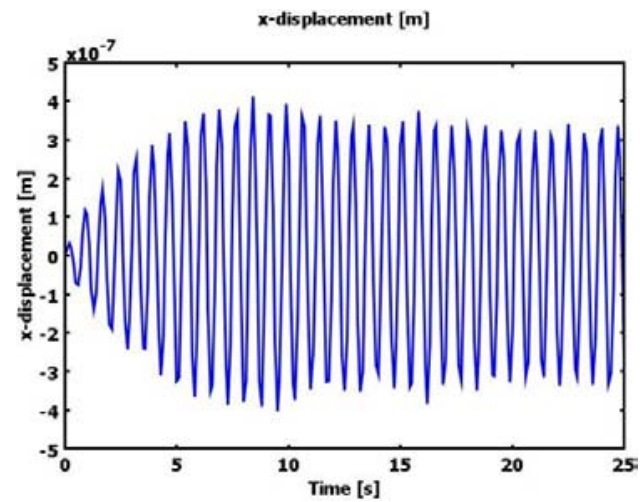

(c)

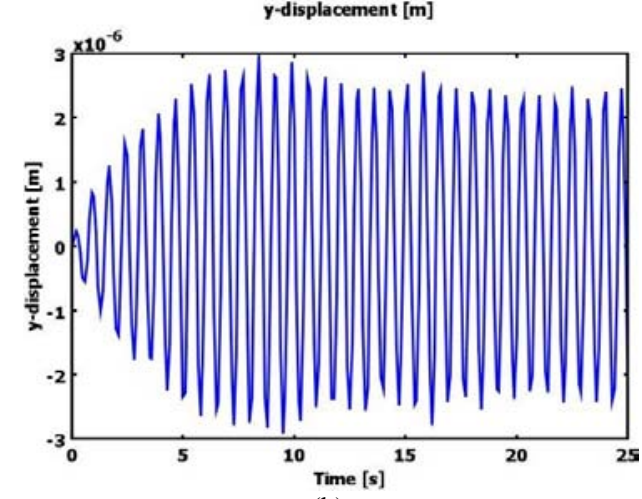

(b)

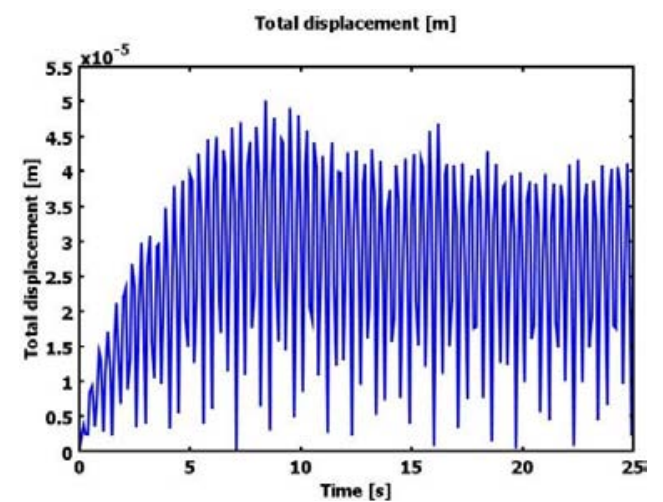

(d)

Figs. 6. PMPG transient analysis displacement during first 35 cycle of time: (a) Z displacement, (b) Y displacement, (c) X displacement, (d) Total displacement 


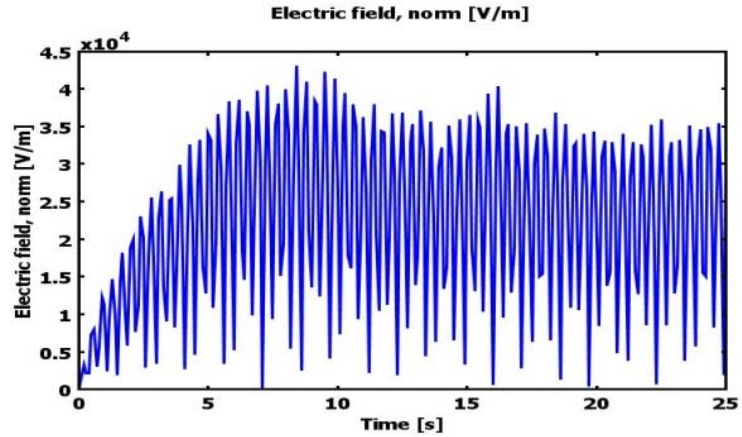

(a)

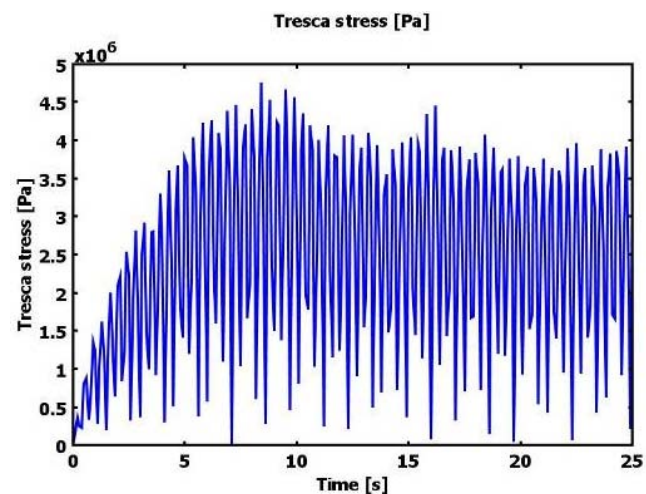

(c)

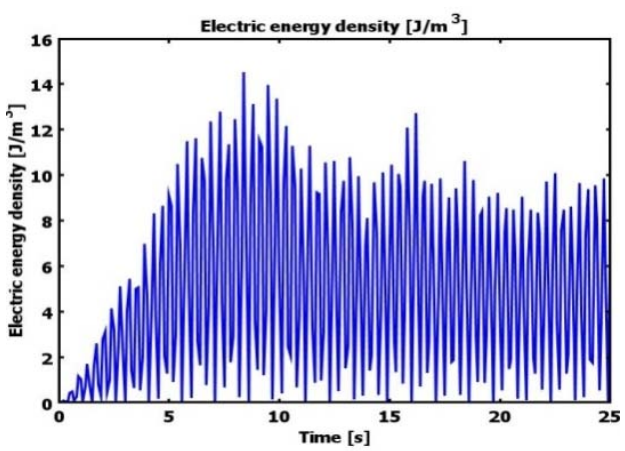

(b)

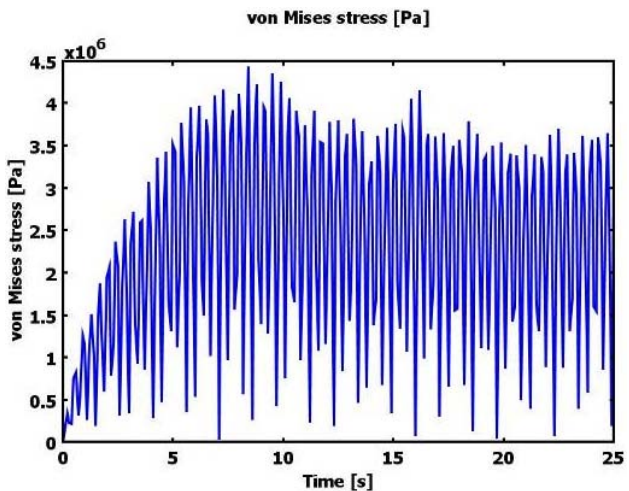

(d)

Figs. 7. PMPG transient analysis during first 35 cycle of time:

(a) Normal electric field, (b) electric energy density, (c) Tresca stress, (d) Von Mises stress

In transient analysis, the PMPG is supplied by using sinusoidal signal amplitude of $0.8 \mathrm{~g}$ at a frequency of 1.35 Hz. Then the behavior of PMPG during the first 25 seconds is studied, which is equal to approximately 35 cycles of time. The device reaches steady-state after approximately 7 seconds and shows stable behavior during that time, which improves the PMPG design and its ability to work probably at this frequency and convert the mechanical energy into electrical energy necessary to small electronic devices. Figs. 6(a)-(d) show the PMPG $\mathrm{Z}, \mathrm{Y}, \mathrm{X}$ displacements, and the total displacement in transient analysis respectively. These figures show that the total displacement is mainly dominated by the Z-axis displacement in the range of $10^{-5} \mathrm{~m}$, while $\mathrm{Y}$ and $\mathrm{X}$ displacements in the range of $10^{-6} \mathrm{~m}$ and $10^{-7} \mathrm{~m}$ respectively. Figs. 7(a) and (b) show the normal electric field and electric energy density, which are produced by PMPG, that is under the study. It can produce up to $4.5 \times 10^{4}$ peak-to-peak $\mathrm{V} / \mathrm{m}$ and $14 \mathrm{~J} / \mathrm{m}^{3}$ of electric charge density. This high value of electric field does not reflect the output power from PMPG that is investigated in this study, but it reflects the power density. The PMPG designs with dimensions in sub millimeters and its thicknesses in micrometers. In case of arranging thousands of PMPG devices in a correct way will result in enough power production to replace the lithium iodide batteries, and open the door in front of producing selfpowered devices at very low frequency, such as cardiac pacemakers or other bio implanted devices operating in a normal body activity around $1 \mathrm{~Hz}$. Figs. 7(c) and (d), respectively, show the maximum stresses and maximum shear stresses that the PMPG can withstand during transient analysis before fracturing, which is about 4.5 $\mathrm{MPa}$. The higher value of stresses in transient analysis compares to frequency analysis is due to the damping coefficient, which is necessary to consider it in transient analysis, while it is negligible in the frequency response analysis.

\section{Conclusion}

In this paper, the L18 orthogonal array is based on the Taguchi optimization method, which is selected with eight control parameters to optimize the quality and damping factor of PMPG design. Taguchi analysis results show that each control parameter affects the quality and damping factor of PMPG by descending order from the highest to the lowest as follows: insulator width, piezoelectric layer width, insulator thickness, proof mass thickness, piezoelectric material, piezoelectric layer thickness, proof mass material, and proof mass length respectively. ANOVA results confirm Taguchi ones where each control parameter affects the quality and damping factor by a different percentage as following: Insulator width $28.19 \%$, piezoelectric layer width $23.24 \%$, insulator thickness $12.65 \%$, proof mass thickness $10.32 \%$, piezoelectric material $10.92 \%$, and piezoelectric layer thickness $7.68 \%$, proof mass material $3.96 \%$, and proof mass length $0.17 \%$. Moreover, the only deviation between Taguchi and ANOVA tests is in proof 
mass thickness and piezoelectric material. The maximum PMPG quality factor is equal to 166.67 and hence the minimum damping factor is equal to 0.003 , something happens in the fifth experiment of the first trial. Taguchi parameter design can successfully verify the optimum parameters obtained at an aluminum proof mass material corresponding to level 1, PZT5A corresponds to level 2 of piezoelectric material, $5 \mathrm{~mm}$ corresponds to level 2 of proof mass length, $2.5 \mathrm{~mm}$ corresponds to level 2 of proof mass thickness, $0.2 \mathrm{~mm}$ corresponds to level 3 for piezoelectric layer width, $60 \mu \mathrm{m}$ corresponds to level 3 of piezoelectric layer thickness, level 1 silicon nitride layer width of $0.12 \mathrm{~mm}$, and $20 \mu \mathrm{m}$ of silicon nitride layer thickness corresponds to level 1. Both Taguchi and ANOVA confirm the same results of determining the parameter and have the most influence on the quality and damping factor for PMPG cantilever beam-based devices that work at very low frequency. The PMPG at optimum quality and lower damping factors are simulated for three basic analyses. The Eigen frequency analysis shows that PMPG works probably in up-down vibrational mode at resonance frequency of $1.35 \mathrm{~Hz}$, which is suitable for cardiac pacemaker applications. The frequency response shows that the PMPG produces maximum electrical field and electric energy density at the first resonance mode in comparison to the other resonance frequencies. In Transient response analysis, the PMPG works probably at the first resonance frequency mode of $1.35 \mathrm{~Hz}$ and reaches the steady state within seven seconds and continues to work smoothly after that. In this paper, PMPG is designed and simulated to work probably at $1.35 \mathrm{~Hz}$, which opens the door widely for fabricating MEMS self-powered devices, which are able to replace lithium iodide batteries into small electronic devices, such as a cardiac pacemaker.

\section{Acknowledgements}

The author would like to thank Abdul Hameed Shoman Foundation (AHSF) for supporting this project under grant $9 / 2018$. The author would like also to thank Al-Hussein Bin Talal University- JORDAN for providing the necessary facilities for this project.

\section{References}

[1] A. Anand and S. Kundu, Design of Mems Based Piezoelectric Energy Harvester for Pacemaker, 2019 Devices for Integrated Circuit (DevIC), Kalyani, India, 2019, pp. 465-469. doi: 10.1109/DEVIC.2019.8783311

[2] D. Chaudhuri, S. Kundu, N. Chattoraj, Design and analysis of MEMS based piezoelectric energy harvester for machine monitoring application, Microsyst. Technol, Vol. 25, (Issue 4), pp 1437-1446, 2019

[3] D. S. Chew, V. Kuriachan, ,Leadless cardiac pacemakers, Current Opinion in Cardiology, vol. 33, (no. 1), pp. 7-13, 2018.

[4] Aabid, M., Elakkary, A., Sefiani, N., Real-Time Cardiac Monitoring Through the Application of an Adaptive Controller to Human Heart, (2017) International Review of Automatic Control (IREACO), 10 (1), pp. 63-71.

doi: https://doi.org/10.15866/ireaco.v10i1.11014

[5] Alrashdan, Mohd H.S., Hamzah, A.A., Majlis, B.Y. Process development of piezoelectric micro power generator for implantable biomedical devices. Micro and Nanosystems. Vol. 7, (Issue 3). 2015.

[6] S. M. Tousif and Z. Çelik-Butler, Array of Linear and Nonlinear Electrostatic Energy Harvesters for Broadband Energy Harvesting, IEEE Sensors Applications Symposium (SAS), Sophia Antipolis, France, 2019, pp. 1-6, 2019.

[7] Yao-Yun Feng, Shih-Jui Chen, Shou-Po Cheng, Development of a miniaturized rotational electromagnetic energy harvester with a liquid metal direct-write process, Sensors and Actuators A: Physical, Vol. 295, Pages 224-230, 2019.

[8] Alrashdan, Mohd H. S., A Hamzah, Azrul Azlan, Majlis, Burhanuddin Yeop, Design and optimization of cantilever based piezoelectric micro power generator for cardiac pacemaker, Microsystem Technologies. Volume 21, (Issue 8), pp 1607-1617. 2014.

[9] Kai Tao, Haiping Yi, Lihua Tang, Jin Wu, Peihong Wang, Nan Wang, Liangxing $\mathrm{Hu}$, Yongqing Fu, Jianmin Miao, Honglong Chang, Piezoelectric $\mathrm{ZnO}$ thin films for 2DOF MEMS vibrational energy harvesting, Surface and Coatings Technology, Volume 359, Pages 289-295, 2019.

[10] Alrashdan, M.H.S., Hamzah, A.A. \& Majlis, B.Y. Power density optimization for MEMS piezoelectric micro power generator below $100 \mathrm{~Hz}$ applications. Microsystem Technologies. 24: 2071. 2018

[11] Alrashdan, M.H.S.; Majlis, B.Y.; Hamzah, A.A.; Marsi, N Design and simulation of piezoelectric micro power harvester for capturing acoustic vibrations, IEEE Regional Symposium on Micro and Nanoelectronics (RSM), 2013. vol., no., pp. 383, 386, 25-27 Sept. 2013

[12] Priya, S., Song, H., Zhou, Y., et al. , A Review on Piezoelectric Energy Harvesting: Materials, Methods, and Circuits. Energy Harvesting and Systems, 4(1), pp. 3-39. Retrieved 30 Sep. 2019.

[13] S. Du, Y. Jia, C. Zhao, G. A. J. Amaratunga and A. A. Seshia, A Nail-size Piezoelectric Energy Harvesting System Integrating a MEMS Transducer and a CMOS SSHI Circuit, in IEEE Sensors Journal. doi: 10.1109/JSEN.2019.2941180

[14] Elahi, H.; Eugeni, M.; Gaudenzi, P. ,A Review on Mechanisms for Piezoelectric-Based Energy Harvesters. Energies, (11), 1850. 2018

[15] Luschi, L., Iannaccone, G., \& Pieri, F. A critical review of reduced one-dimensional beam models of piezoelectric composite beams. Journal of Intelligent Material Systems and Structures, 30(8), 1148-1162. 2019.

[16] Taguchi G . Hocheng Taguchi methods orthogonal arrays and linear graphs, tools for quality engineering. (American Supplier Institute. 1987. Dearborn, pp 35-38.)

[17] Taguchi G., Introduction to quality engineering. (Asian Productivity Organization. 1990. Tokyo.)

[18] Park, S., Kim, H., Kim, J. et al., Taguchi Design of PZT-Based Piezoelectric Cantilever Beam with Maximum and Robust Voltage for Wide Frequency Range Journal of Electronic Materials pp 1-9. 2019. doi: https://doi.org/10.1007/s11664-019-06994-1

[19] Lee, L.T., Mohamed, M.A., Yahya, I. et al. Comparison of piezo electric energy harvesting performance using silicon and graphene cantilever beam, Microsyst Technol Volume 24, (Issue 9), pp 3783-3789. 2018

[20] Jui-Ching Hsieh, David T.W. Lin, Chia-Li Lin,The development and optimization of an innovative piezoelectric energy harvester on the basis of vapor-induced vibrations, Mechanical Systems and Signal Processing, Volume 131, Pages 649-658. 2019.

[21] Doan, A.T., Yokoyama, T., Dao, T.D., Ishii, S., Ohi, A., Nabatame, T., Wada, Y., Maruyama, S., Nagao, T. A MEMSBased Quad-Wavelength Hybrid Plasmonic-Pyroelectric Infrared Detector, Micromachines, 10, (413), 2019.

[22] Houssini, M., Ahmad, A., El-Bazzal, Z., Hamieh, H., Noun, Z., Conception of a Malleable Multiband Antenna Using RF MEMS Varactors for WIMAX802.16d and X Band, (2017) International Journal on Communications Antenna and Propagation (IRECAP), 7 (2), pp. 147-153. doi: https://doi.org/10.15866/irecap.v7i2.11828

[23] Kaddari, M., Ennawaoui, C., El Mouden, M., Hajjaji, A., Semlali, 
A., Diagnosis of Shaft Misalignment Fault by Piezoelectric Materials to Improve Reliability of Induction Motors, (2019) International Journal on Engineering Applications (IREA), 7 (4), pp. 137-144.

doi: https://doi.org/10.15866/irea.v7i4.17653

[24] Mendoza Casseres, D., Valencia, G., Duarte Forero, J., InCylinder Pressure Signal Processing Applied to the Diagnosis of Combustion in Low Displacement Diesel Engine, (2020) International Journal on Communications Antenna and Propagation (IRECAP), 10 (2), pp. 137-144.

doi: https://doi.org/10.15866/irecap.v10i2.18640

\section{Authors' information}

Department of Electrical Engineering, college of Engineering, AlHussein Bin Talal University, Ma' an 71111, Jordan.

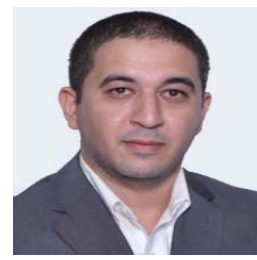

Mohd Alrashdan. DAIR ABI SAID, April 1, 1983, received the B.S. degrees in Biomedical Engineering from Jordan University of Science And Technology in 2006.received M.S and PhD degree in Electrical engineeringMicroelectronics from National University of Malaysia (UKM) in 2007 and 2016 respectively. From 2016 until present, he worked as a full time lecturer in Al- Hussein Bin Talal University - Maán , Jordan. During 2007-2016, he finished his $\mathrm{PhD}$ and focused in modelling, simulation, and fabrication of piezoelectric micro power generator for low frequency application using MEMS technology, during 2016present he works as a full time lecturer and focused in power electronics and power systems analysis. Dr. Mohd Alrashdan is a member in Jordan engineers association, cooperate with institute of micro engineering and nano electronics

E-mail: moh.alrashdan@ahu.edu.jo 